\title{
Dynamic projection on Feshbach molecules: a probe of pairing and phase fluctuations
}

\author{
Ehud Altman ${ }^{1}$ and Ashvin Vishwanath ${ }^{2}$ \\ ${ }^{1}$ Physics Department, Harvard University, Cambridge, MA 02138. \\ ${ }^{2}$ Department of Physics, University of California, Berkeley, CA 94720;
}

\begin{abstract}
We describe and justify a simple model for the dynamics associated with rapid sweeps across a Feshbach resonance, from the atomic to the molecular side, in an ultra cold Fermi system. The model allows us to relate the observed molecule momentum distribution, including its dependence on the sweep rate, to equilibrium properties of the initial state. For initial state near resonance, we find that phase fluctuations sharply reduce the observed condensate fraction. Moreover, for very fast sweeps and low temperatures, we predict a surprising nonmonotonic dependence of the molecule condensate fraction on detuning, that is a direct signature of quantum phase fluctuations. The dependence of the total molecule number on sweep rate is found to be a sensitive probe of pairing in the initial state, whether condensed or not. Hence it can be utilized to establish the presence of a phase fluctuation induced 'pseudogap' phase in these systems.
\end{abstract}

Experiments with ultra cold fermions near a Feshbach resonance (FR), opened a new window to the study of strongly interacting condensates 1, 2, 3]. They can access the strongly coupled regime intermediate between weak pairing BCS superfluidity and a BEC of molecules. In this region, deviations from mean field theory a la $B C S$ [4, 5, 6], are expected to be large. In addition, the ability to rapidly vary the interaction parameters, provides a unique opportunity to study quantum dynamics far from equilibrium [7, 8, 9]. The focus of recent experiments has been to utilize dynamics, in this case rapid magnetic field sweeps across the FR, in order to probe the equilibrium properties of the condensate in the crossover region [2, 3]. The idea was to convert cooper pairs in the initial state, which would otherwise unbind during free expansion, into molecules. The fact that the molecule momentum distribution depended on the start position has been offered as strong indication that indeed properties of the initial equilibrium state were being accessed. However, precise connections to such properties are lacking.

In this letter we formulate an approach that allows us to relate equilibrium properties of such strongly interacting fermions to the measurements in the dynamical experiments. Our approach targets the regime of 'fast' magnetic field sweeps. Roughly, these are ramp rates at which the conversion efficiency of atoms into molecules changes significantly with changing ramp rate. Empirically in the ${ }^{40} \mathrm{~K}$ system this implies a ramp rate faster than $1 / 20 G / \mu \sec 2$ ], while it was too fast to be accessed in $\left.{ }^{6} \mathrm{Li} 3\right]$. The physics in this regime turns out to have a remarkable simplicity, and for this reason we will focus on it in this letter, although the bulk of experiments to date have been done at slower ramp speeds. It is hoped that the results of the present study will stimulate experimental investigation of this regime.

Consider for a moment the extreme limit of an infinitely fast sweep. Then, we can apply the sudden approximation and simply use the initial state to evaluate the final population of molecules. This intuitive picture was advocated by Regal et al[2] (see also [10]). Using

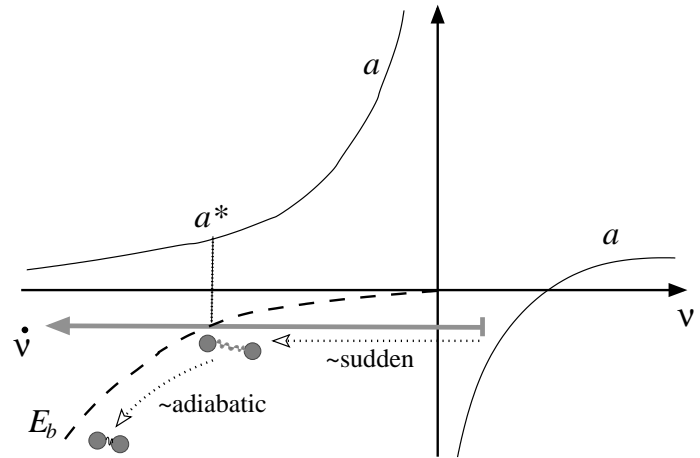

FIG. 1: Model dynamics. Field sweep is effectively sudden up to detuning $\nu_{\star}$ depending on the sweep rate $\dot{\nu}$. At this point the scattering length (and molecule size) is $a_{\star}$. The molecule is assumed to evolve adiabatically from there.

the same assumption, Diener and Ho 11 estimated the fraction of condensed molecules as a function of the detuning of the initial state from resonance. The molecule momentum distribution in this approach is given by

$$
n_{m}(\mathbf{q})=\left\langle b_{\mathbf{q}}^{\dagger} b_{\mathbf{q}}\right\rangle_{i} \quad b_{\mathbf{q}}^{\dagger}=\int d \mathbf{k} \varphi_{f}(k) c_{\frac{\mathbf{q}}{2}+\mathbf{k} \uparrow}^{\dagger} c_{\frac{\mathbf{q}}{2}-\mathbf{k} \downarrow}^{\dagger},
$$

where $\varphi_{f}(k)$ is the molecular WF at the end of the ramp, deep in the molecular side of the resonance. The average \langle\rangle$_{i}$ is taken over the initial state, which in Ref. 11] was assumed to be a BCS WF, calculated within the mean field theory of [5]. Indeed, this gives a bimodal momentum distribution, with a peak at $\mathbf{q}=0$ due to cooper pairs projected to molecules; as well as molecules at $\mathbf{q} \neq 0$ due to pairwise projection of atoms belonging to different cooper pairs. Although this is roughly what is seen in the experiments, it is unsatisfactory in two important respects. (i) Mean field theory is used to calculate the initial state, whereas it is well known that in the crossover regime near the resonance, quantum and thermally induced phase fluctuations play a very important role. (ii) The approach ignores the dynamical aspects of the experiment and is by definition unable to predict the 
dependence of measured quantities (eg. conversion efficiency and molecular condensate fraction) on ramp rate.

Both these issues are addressed in this paper. The first, by going beyond the mean field theory, using the RPA [13] to include Gaussian phase fluctuations (or "non condensed cooper pairs"). In the BCS to BEC crossover regime these sharply reduce the observed condensate fraction relative to the mean field result of Ref. [11], even at zero temperature. For fast sweeps at low temperature this leads us to predict a surprising non monotonic behavior of the molecule condensate fraction versus detuning of the initial state from resonance. Point (ii) raised above is addressed by an effective model for the dynamics. For fast sweeps we argue that the time evolution of the system can be approximated by a 'sudden' part followed by an 'adiabatic' time evolution part. The point at which the time evolution changes character depends on the ramp rate, the sudden evolution persisting for longer at higher ramp rates. Thus, the dynamics is approximated by projecting the initial state of the system onto an effective molecular WF, which is determined by the ramp rate. So, we will be using Eq. (1), but with the effective molecular WF, $\varphi$ that depends on the ramp rate. In this way we are able to obtain the parametric dependence of various measured quantities, such as conversion efficiency into molecules and molecular condensate fraction on the ramp rate, and the parameters of the initial state. At these fast sweep rates, we find that conversion of Cooper pairs into molecules is vastly more efficient than that of uncorrelated pairs of atoms. This applies both to condensed and uncondensed Cooper pairs, and ultimately results from a short distance singularity present in the Cooper pair wavefunction (WF), that allows them to have a non-negligible overlap even with small sized molecules. Since these dynamical measurements are equally sensitive to noncondensed Cooper pairs, they can potentially probe the phase fluctuation induced pseudogap phase (where thermal and quantum phase fluctuations destroy the condensate but pairing remains). Details such as the momentum distribution of noncondensed pairs may also be accessed.

We now motivate the model dynamics via physical arguments and derive the consequences for the system of interest. Later we perform a nontrivial check by showing that this simple scheme indeed reproduces the physics in a nontrivial toy model (the Dicke model) which is solved numerically without approximations. In what follows we concentrate on a wide $\mathrm{FR}$, relevant to experiments in ${ }^{40} K[2]$ and ${ }^{7} L i\left[3\right.$ ? ]. That is, $g_{s} \equiv g \sqrt{n / 2}>>\epsilon_{f}$ where $g$ is the coupling between the open and closed channels and $n$ is the atom density. For most purposes it is then possible to neglect the occupation of closed channel molecules. The problem reduces to spin- $\frac{1}{2}$ fermions interacting via a contact potential

$$
H=\int d x c_{\sigma}^{\dagger}(x)\left(-\frac{\nabla^{2}}{2 m}-\mu\right) c_{\sigma}(x)-u c_{\uparrow}^{\dagger}(x) c_{\downarrow}^{\dagger}(x) c_{\downarrow}(x) c_{\uparrow}(x)
$$

Ultraviolet divergences are avoided in the standard fashion by writing all physical results in terms of an s-wave scattering length $a$ instead of $u$. Near resonance the scattering length diverges as $a \approx-m g^{2} / 4 \pi \nu$ where $m$ is the atomic mass, and $\nu$ is the detuning in energy units, which is related to the magnetic detuning via the magnetic moment difference $\Delta \mu$ between closed and open channels: $\nu=\Delta \mu\left(B-B_{0}\right)$.

Our two stage approximation of the dynamics is depicted graphically in Fig. 1] For slow sweeps, atoms are converted adiabatically to weakly bound Feshbach molecules (FM) [14]. The binding energy of these molecules is $E_{b}=-1 /\left(m a^{2}\right) \propto \nu^{2}$ (for $k_{f} a<<1$ ). Now consider a change in the detuning parameter at a constant rate $\dot{\nu}$. Once the binding energy is large enough, such that $\dot{E}_{b} \ll E_{b}^{2}$, the time evolution is expected to be adiabatic. So, for a given sweep rate $\dot{\nu}$, there is a characteristic detuning $\nu_{\star}$, which marks a dynamic crossover for the system. At $\nu_{\star}, \dot{E}_{b} \approx E_{b}^{2}$ [12]. Our simplified two stage model for the dynamics approximates the change up to $\nu_{\star}$ as sudden, while the rest is considered as perfectly adiabatic. Thus the WF at the initial state is effectively "projected" on FMs that occur at detuning $\nu_{\star}$. These molecules evolve adiabatically into more tightly bound ones corresponding to the final value of the field, while the rest of the atomic population remains unbound.

Within this model, a faster sweep rate simply moves the break point $\nu_{\star}$ to larger negative detuning, leading to effective projection of the initial WF on smaller FMs. We can establish a precise connection between the sweep rate and the size of the effective molecule WF. The typical size of the FM at $\nu_{\star}$ is the scattering length $a_{\star}\left(\nu_{\star}\right)$. Using $E_{b}=-1 /\left(m a^{2}\right)$, and the relation between the scattering length and the detuning $\nu$ quoted earlier, with the break condition $E_{b}^{2}\left(a_{\star}\right)=d E_{b}\left(a_{\star}\right) / d t$ we obtain

$$
k_{f} a_{\star}=\left(\frac{3 \pi}{4} \frac{g_{s}^{2}}{\dot{\nu}}\right)^{1 / 3}
$$

We now employ the two stage model and Eq. (3) to determine the main features of the final molecule distribution and its dependence on both initial state and sweep rate. We need to evaluate the following correlation function, in the initial state:

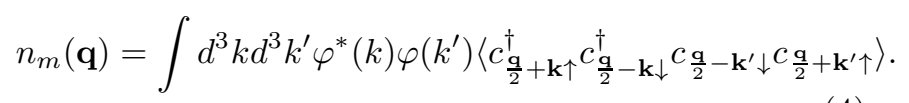

The size of the molecular pair WF $\varphi(k)$ is $a_{\star}$, as prescribed by the model dynamics. First consider a mean field (BCS) approximation of the initial state. In this 
case the correlation function appearing in (4) may be factorized to obtain, as in Ref. [11]:

$$
\begin{aligned}
n_{m}(\mathbf{q})= & \left|\int d^{3} k \varphi(k)\left\langle c_{\mathbf{k} \uparrow}^{\dagger} c_{-\mathbf{k} \downarrow}^{\dagger}\right\rangle\right|^{2} \delta(\mathbf{q}) \\
& +\int d^{3} k|\varphi(k)|^{2}\left\langle n_{\frac{\mathbf{q}}{2}+\mathbf{k} \uparrow}\right\rangle\left\langle n_{\frac{\mathbf{q}}{2}-\mathbf{k} \downarrow}\right\rangle
\end{aligned}
$$

The first contribution, proportional to the anomalous expectation value, gives the condensed part of the distribution. In fact this is just the square of the overlap between the final molecule WF and the "cooper pair WF". The second, non condensed part, contains the normal expectation values. To make further progress we note that if the molecule size $a_{\star}$ is much smaller than the interparticle spacing, we can replace the exact WF with a box WF in momentum space, of the same spatial extent. We take $\varphi(\mathbf{k})=\sqrt{3 / 4 \pi} a_{\star}^{3 / 2}$ for $k<1 / a_{\star}$ and $\varphi(\mathbf{k})=0$ outside this sphere. Now $\varphi(\mathbf{k})$ serves as a cutoff to the relative momentum integrals. Using this in (5), the number of normal molecules is found to be

$$
N_{n}=(N / 2)\left(k_{f} a_{\star}\right)^{3}
$$

where $N$ is the total atom number. This result is easily understood in terms of the overlap of a random pair of atoms in the Fermi sea, with a molecular WF. The conversion efficiency is then proportional to the ratio of the molecule volume to that occupied on average by nearby atoms. In contrast, the number of condensed molecules calculated from (5) is

$$
N_{0}=\frac{6 V a_{\star}^{3}}{(2 \pi)^{2}}\left|\int_{0}^{a_{\star}^{-1}} \frac{d k k^{2} \Delta / 2}{\sqrt{\Delta^{2}+\xi_{k}^{2}}}\right|^{2}=\frac{9 N}{8}\left(\frac{\Delta}{\epsilon_{f}}\right)^{2} k_{f} a_{\star}
$$

The last step relies on the high momentum divergence of the integral, due to which it depends crucially on the high momentum cutoff. The result (7) will have important consequences on interpretation of experiments, and it is worthwhile to remark on its physical origin. The number of condensed molecules is proportional to the square of the overlap of the molecular WF $\varphi_{m}$ with the Cooper pair $\mathrm{WF} \varphi_{c}$. Now, the molecule WF is appreciable only within a region $r<a_{\star}$, where $\varphi_{m}(r) \sim a_{\star}^{-3 / 2}$. Since this region is smaller than the average inter-particle distance, the molecules probe the Cooper pairs at very short distances. Now, the Cooper pair WF has a singular short distance behavior, $\varphi_{c}(r) \propto 1 / r$. Therefore $\left|\left\langle\varphi_{m} \mid \varphi_{c}\right\rangle\right|^{2} \propto a_{\star}$ which is a much larger overlap than might be naively expected. Note, in solid state systems where a natural short distance cutoff exists, these features are absent and hence have not been emphasized.

Combining the mean field results for $N_{0}$ and $N_{n}$ we can evaluate the condensate fraction in the molecule distribution:

$$
f_{M F}=\frac{N_{0}}{N_{0}+N_{m}}=\frac{1}{1+4 / 9\left(\epsilon_{f} / \Delta\right)^{2}\left(k_{f} a_{\star}\right)^{2}}
$$

The dependence on the initial state enters this expression through the factor $\epsilon_{f} / \Delta$. For large positive detuning we expect weak pairing $\Delta / \epsilon_{f} \sim e^{-1 / k_{f} a}$, while close to resonance $\Delta \sim \epsilon_{f}$. For sufficiently fast sweep rates, $k_{f} a_{\star}<<1$ we have $f_{M F} \sim 1$ in the vicinity of the resonance. This is because Cooper pairs are much more efficiently converted into molecules, and within the mean field approximation used above, all Cooper pairs are condensed. However, especially in this region close to the resonance we expect phase fluctuations (Cooper pairs at finite momenta) to be excited, which will lead to $f<1$.

To obtain such corrections to the mean field result (5) we calculate the correlation function (44) within the RPA approximation. The details of the calculation are left to 12], here we briefly outline the main steps and the results. Following Ref. [13], we consider a path integral representation of the partition function $\mathcal{Z}$ determined by the Hamiltonian 2 The interaction term may be decoupled with a Hubbard-Stratanovitch pair field $\Delta(\mathbf{q}, \omega)$. In order to extract the desired correlation functions, we introduce a source term $J(\mathbf{q}, \omega) \int d^{4} k \varphi(k) c_{\frac{\mathbf{Q}}{2}+\mathbf{K}}^{\dagger} \uparrow c_{\frac{\mathbf{Q}}{2}-\mathbf{K}^{\downarrow}}^{\dagger}+$ h.c., where we have used the four vector notation $\mathbf{Q}=$ $(\mathbf{q}, \omega)$. At $T>0$ the integral over imaginary frequencies is converted into the usual Matsubara sum. Then, the desired molecular distribution function is:

$$
n_{m}(\mathbf{q})=\frac{1}{\mathcal{Z}} \sum_{\omega \omega^{\prime}} \frac{\delta^{2} \mathcal{Z}}{\delta J(\mathbf{q}, \omega) \delta J^{\star}\left(\mathbf{q}, \omega^{\prime}\right)}
$$

While the saddle point approximation gives us the BCS result, here we go beyond and expand $\Delta(\mathbf{q}, \omega)=$ $\Delta_{0} \delta(\mathbf{Q})+\eta(\mathbf{q}, \omega)$, where $\Delta_{0}$ is the saddle-point value of the gap. The RPA approximation involves integrating out the fermions and expanding the resulting effective action to quadratic order in the $\eta$ and $J$ fields. The poles in the $\eta$ propagator give the collective mode spectrum. Finally, integrating out the $\eta$ fields gives us an effective action solely in terms of $J$ from which the molecular distribution function $n_{m}(\mathbf{q})_{M F}+\delta n_{m}(\mathbf{q})(\delta n(\mathbf{q})$ is the contribution due to fluctuations) may be evaluated.

Assuming that the fluctuation contribution is dominantly from superfluid phonons, we can make a small momentum, small frequency expansion, where the $J$ propagator takes the form $F\left(k_{f} a_{\star}, k_{f} a\right)\left[(c q)^{2}-(i \omega)^{2}\right]^{-1}$, where $c$ is the sound velocity in the superfluid and the function $F$ will be discussed shortly. It is important to note that the implied linear dispersion is only an approximation due to the low $q$ and $\omega$ expansion. At momenta $q \gtrsim 4 m c$ the dispersion becomes quadratic. In addition at $\omega>\Delta$ phase fluctuations can decay into quasiparticle excitations, leading to damping in the BCS limit at $q \gtrsim \Delta / c \approx 1 / \xi$

Carrying out the Matsubara summation we obtain the RPA correction to the molecule momentum distribution

$$
\delta n(\mathbf{q})=F\left(k_{f} a_{\star}, k_{f} a\right) \frac{\operatorname{coth}(c q / 2 T)}{2 c q} .
$$




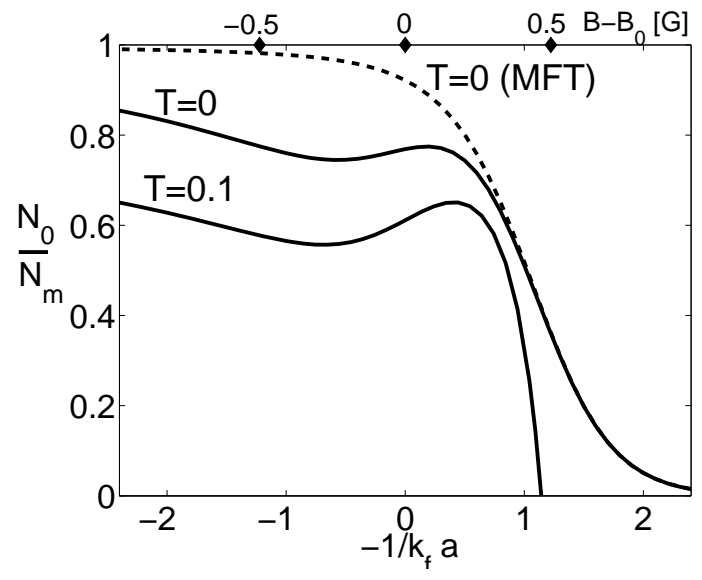

FIG. 2: Calculated molecule condensate fraction versus $-1 / k_{f} a$ in the initial state (bottom axis), and versus magnetic field detuning for parameters of ${ }^{40} \mathrm{~K}[2]$ (top axis). The sweep rate corresponds to $k_{f} a_{\star}=0.3$. Solid lines include the effect of quantum and thermal phase fluctuations. Dashed line shows the $(T=0)$ mean field result for comparison.

The number of non-condensed molecules due to these fluctuations is found by integrating over $\mathbf{q}$ up to a natural cutoff. As discussed above, such a cutoff is provided in the BCS limit by $q_{1}=1 / \xi$, and in the BEC limit by $q_{2}=4 m c$. To cover the whole range we use the cutoff $q_{0}^{-1}=q_{1}^{-1}+q_{2}^{-1}$ which interpolates between the two limits.

The variation of the above fluctuation correction with sweep rate is encoded into the dependence of the function $F\left(k_{f} a_{\star}, k_{f} a\right)$ on $k_{f} a_{\star}$. The leading dependence on $k_{f} a_{\star}$ is found to be linear, which arises from the fact that non-condensed Cooper pairs are as efficiently transformed into molecules as condensed Cooper pairs. By contrast, conversion of unpaired atoms into molecules is much less efficient and scales as $\left(k_{f} a_{\star}\right)^{3}$.

The full function $F\left(k_{f} a_{\star}, k_{f} a\right)$ can be computed along the crossover from BCS to BEC 12]. Fig. 2] depicts the calculated condensed molecule fraction including fluctuation corrections for sweep rate corresponding to projection at $k_{f} a_{\star}=0.3$. The nonmonotonic dependence on detuning toward the BCS limit is due to competition between two effects. On the one hand decreasing phase fluctuations act to increase the condensed molecule fraction. On the other hand the ratio $\epsilon_{f} / \Delta$ appearing in the normal atom contribution diverges exponentially at large detuning and eventually leads to vanishing of the molecular condensate fraction.

We now turn to a non trivial check of the two stage approximation of the dynamics. Based on this model we found enhanced conversion efficiency at rapid sweeps of cooper pairs (both condensed and non condensed) into FMs, compared to unpaired atoms. Using (3), and the linear scaling in $k_{f} a_{\star}$ of the number of molecules arising from cooper pairs, we obtain a parametric dependence
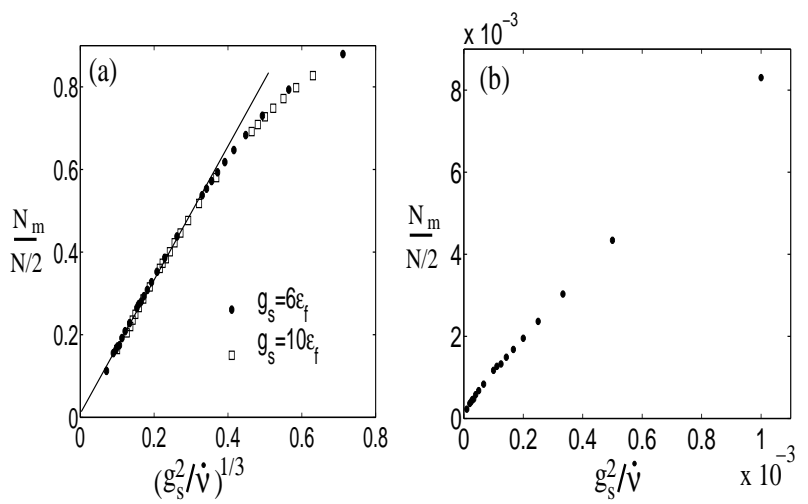

FIG. 3: Simulated mean Dicke model dynamics: Final molecule number as a function of the sweep rate. (a) Initial state close to resonance (strong pairing). The curves for different coupling constants $g_{s}$ fall on a universal curve $\propto 1 / \dot{\nu}^{1 / 3}$ at fast rates, in agreement with (3) and (7). (b) Initial state far detuned $\left(\nu=400 \epsilon_{f}, g_{s}=6 \epsilon_{f}\right)$, i.e. weak pairing. The $1 / \dot{\nu}$ behavior due to (6) dominates.

on the sweep rate $\propto\left(g_{s}^{2} / \dot{\nu}\right)^{1 / 3}$. By contrast, from (6) and (3), the conversion efficiency for unpaired atoms is much lower, $\propto g_{s}^{2} / \dot{\nu}$. We now wish to verify these nontrivial dependences using a numerical simulation of the dynamics, not relying on the two-stage model. We start from the microscopic two channel Hamiltonian [ 6 ]

$$
\begin{aligned}
H= & \sum_{\mathbf{k} \sigma}\left(\epsilon_{\mathbf{k}}-\mu\right) c_{\sigma \mathbf{k}}^{\dagger} c_{\sigma \mathbf{k}}+\sum_{\mathbf{k}}\left(\epsilon_{k} / 2+\nu-2 \mu\right) m_{k}^{\dagger} m_{k} \\
& -g \sum_{\mathbf{k q}}\left(m_{\mathbf{q}} c_{\uparrow \mathbf{k}}^{\dagger} c_{\downarrow \mathbf{q}-\mathbf{k}}^{\dagger}+H . c .\right)+\mu N,
\end{aligned}
$$

which is regularized by absorbing the high momentum cutoff into renormalized detuning parameter [6]. Note, $m_{\mathbf{q}}$ describes a (bare) closed channel molecule.

We compute the dynamics of (11) within the Dickie model (i.e. keeping only $m_{\mathbf{q}=0}$ ). The initial state is taken to be the equilibrium solution at detuning $\nu \geq 0$. Then $\nu$ is changed at a constant rate to far negative detuning where the equilibrium population of $m_{0}$ would be $\sim 96 \%$ and the resulting dynamics (see e.g. [8]) is calculated numerically with no approximation. Following the experiments we count the number of molecules in the final state, which to a good approximation is $\left|\left\langle m_{0}\right\rangle\right|^{2}$. According to the two stage model this is directly related to the number of FMs produced after the sudden stage.

The results are summarized in Fig. 3] Most importantly, for initial state at resonance (i.e. strong pairing), the dependence on sweep rate fits $1 / \dot{\nu}^{1 / 3}$, as expected from our two stage model. By contrast, for weak pairing, deep in the BCS regime the normal contribution $1 / \dot{\nu}$ dominates (Fig. 3 3 ). The strong suppression of the prefactor of $1 / \dot{\nu}$ is an artifact of the Dickie model, which prohibits occupation of molecules with $q \neq 0$.

In summary, we presented a simple model of the dynamics of rapid magnetic field sweeps across the FR. This 
allowed to relate the measurements of such dynamical experiments to properties of the initial equilibrium state of the Fermions. For rapid sweeps at low temperatures in the crossover region we predict a a non monotonic behavior of the final condensed molecule fraction versus detuning, which is a direct signature of the quantum phase fluctuations in the initial state. In addition, the conversion efficiency to molecules at fast sweep rates is argued to be a sensitive probe of pairing, whether in a condensed state or not. This can be used to establish the presence of a fluctuation induced pseudogap phase. In this regime we expect enhanced conversion efficiency due to the presence of noncondensed fermion pairs, despite a vanishing condensate fraction.

Acknowledgements. Discussions with E. Demler, M. Greiner, D. Jin, W. Ketterle, D. Petrov, C. Regal, and M. Zwierlein are gratefully acknowledged. A.V. would like to thank the A. P. Sloan foundation for support.

1] K. M Ohara et al, Science 298, 2179 (2002); K. Strecker et al, Phys. Rev. Lett. 91, 080406 (2003); T. Bourdel et al, Phys. Rev. Lett. 91, 020402 (2003); M. Bartenstein et al, Phys. Rev. Lett. 92, 120401 (2004);

[2] C. A. Regal, M. Greiner, and D. S. Jin, Phys. Rev. Lett. 92, 040403 (2004).

[3] M. Zwierlein, et.al., Phys. Rev. Lett. 92, 120403 (2004)

[4] D.M. Eagles, Phys. Rev. 186, 456 (1969).

[5] A.J. Leggett, J. Phys. (Paris), Colloq. 41, 7 (1980);

[6] J. N. Milstein, S. J. J. M. F. Kokkelmans, and M. J. Holland Phys. Rev. A 66, 043604 (2002).

[7] R. A. Barankov, L. S. Levitov, and B. Z. Spivak, Phys. Rev. Lett. 93, 160401 (2004); R. A. Barankov and L. S. Levitov, Phys. Rev. Lett. 93, 130403 (2004).

[8] A. V. Andreev, V. Gurarie, and L. Radzihovsky, Phys. Rev. Lett. 93, 130402 (2004)

[9] V. M. Galitzki, cond-mat/0404125

[10] G. M. Falco and H. T. C. Stoof, cond-mat/0402579

[11] Roberto B. Diener and Tin-Lun Ho, cond-mat/0404517

[12] E. Altman and A. Vishwanath, in preparation.

[13] J. R. Engelbrecht, M. Randeria, and C. A. R. Sa de Melo Phys. Rev. B 55, 15153 (1997).

[14] C. A. Regal, C. Ticknor, J. L. Bohn, and D. S. Jin, Nature 424, 47 (2003).

[15] M. Greiner, C. A. Regal, and D. S. Jin, Nature 426, 537 (2003). 